\title{
Información médica sobre el cáncer de mama y repercusiones psicológicas en pacientes oncológicos provenientes de España durante el año 2011
}

Sheila Pintado*

\begin{abstract}
*Doctora en Psicología. Universidad de las Américas Puebla (UDLAP). Ex Hacienda Sta. Catarina Mártir. San Andrés Cholula. Puebla. México Correspondencia: Doctora Sheila Pintado. Correo electrónico: maria.pintado@udlap.mx
\end{abstract}

\section{RESUMEN}

Introducción: la falta de información médica proporcionada puede resultar perjudicial para el funcionamiento psicológico del paciente oncológico, pudiendo presentarse síntomas de ansiedad y depresión ante esta necesidad no cubierta. Objetivo: analizar la relación entre los niveles de información médica sobre el diagnóstico, el pronóstico, el tratamiento y los síntomas de ansiedad y depresión en una muestra de pacientes con cáncer de mama. Materiales y métodos: se trató de un estudio transversal con 131 mujeres con cáncer de mama a las que se les aplicó una entrevista semiestructurada para recabar datos sociodemográficos y clínicos, y los niveles de información médica respecto a la enfermedad, así como la Escala Hospitalaria de Ansiedad y Depresión. La muestra fue no probabilística de cuatro hospitales públicos de España durante el año 2011. Los análisis estadísticos se realizaron mediante el programa estadístico SPSS. Resultados: los datos obtenidos muestran que la falta de información sobre el diagnóstico de la enfermedad, el pronóstico y el tratamiento junto con la incomprensibilidad de la información estaban relacionados con síntomas depresivos y malestar emocional general. Sin embargo, no se encontraron relaciones significativas entre estas variables y la ansiedad. Conclusiones: la información que reciben las pacientes con cáncer de mama sobre su enfermedad, el pronóstico y el tratamiento médico influye en su bienestar emocional, presentándose mayores niveles de depresión en las pacientes que tienen escasa información, y esto es importante que sea tenido en cuenta por los profesionales médicos. MÉD.UIS. 2017;30(3):13-20.

Palabras clave: Neoplasias de la Mama. Comunicación. Depresión. Ansiedad.

\section{Medical information on breast cancer and psychological repercussions in cancer patients from Spain during 2011}

\section{ABSTRACT}

Introduction: the lack of medical information can be harmful for the psychological adjustment of oncological patients, as symptoms of anxiety and depression could appear. Objective: to analyze the relationship between the levels of medical information about the diagnosis, prognostic and treatment, and symptoms of anxiety and depression in a sample of patients with breast cancer. Materials and method: cross-sectional study with 131 women with breast cancer who were assessed by a semi structured interview to evaluate the sociodemographic and clinical data and levels of medical information. In addition, The Hospital Anxiety and Depression Scale was used. The participants were recruited by non-probability sampling in four public hospitals of Spain. The statistical analysis were realized through SPSS. Results: the data obtained showed that the lack of information about the diagnosis, prognosis and treatment was related to symptoms of depression and poor general emotional well-being. Moreover, the incompressibility of the medical information was related to depression. However, the symptoms of anxiety were not statistically significant between these variables. Conclusions: the medical information that breast cancer patients receive about their illness, the prognosis and treatment affects emotional well-being and this is important to be considerate by medical team. MÉD.UIS. 2017;30(3):13-20.

Keywords: Breast Neoplasms. Communication. Depression. Anxiety

¿Cómo citar este artículo?: Pintado S. Información médica sobre el cáncer de mama y repercusiones psicológicas en pacientes oncológicos provenientes de España durante el año 2011. MÉD.UIS. 2017;30(3):13-20. 


\section{INTRODUCCIÓN}

Informar a los pacientes sobre el diagnóstico de cáncer y su pronóstico es una tarea compleja y difícil'. El diagnóstico de cáncer presenta habitualmente una connotación negativa asociada a sufrimiento y muerte ${ }^{2}$. Asimismo, la cultura juega un rol importante en la manera en que vemos el cáncer y en el proceso de entrega de información médica. En este sentido, en algunos grupos culturales se considera ético y necesario mantener al paciente informado sobre el diagnóstico y pronóstico de su enfermedad; sin embargo, en otros grupos esto se considera cruel y se tiene la idea de que proveer información al paciente puede acelerar su sufrimiento y agravar la enfermedad ${ }^{3}$.

Por ejemplo, en una investigación que se llevó a cabo en España por Trill (2003), con el objetivo de evaluar la percepción respecto a la información médica recibida en 591 pacientes con cáncer de próstata, se encontró que el 90,2\% de los pacientes afirmó haber obtenido una correcta cantidad de información sobre su enfermedad. Además, el 70,3\% aceptó mejor la enfermedad debido a la información que recibió y el 60,5\% dijo tener una mejor capacidad de resolución de problemas gracias a ello4. Sin embargo, hay países en los cuales el diagnóstico de cáncer se comunicaba a menos del $50 \%$ de los pacientes ${ }^{3}$.

En otro estudio realizado en España por Peiró y cols. (2006) donde se entrevistaron pacientes con diversos tipos de cáncer (mama, pulmón, endometrio, ovario y colon) y se pretendía conocer la información que tenían los pacientes sobre su enfermedad antes de la primera entrevista con el oncólogo, se observó que de los 105 pacientes evaluados, el 58,1\% tenía algún tipo de información más o menos precisa, mientras que el $41,9 \%$ no tenía ninguna información clara por parte del facultativo remitente sobre el motivo de su derivación. $Y$ respecto al tipo de información que se recibió, de este 58,1\% ( $n=61), 39$ de ellos había sido informado de manera imprecisa (como por ejemplo, el médico les dijo que "había aparecido una mancha en las pruebas en determinado órgano") y los otros 22 pacientes sí tenían una información clara sobre la existencia de un tumor maligno o cáncer. Por tanto, en este estudio se pudo concretar que solamente el 20,95\% de los pacientes tenía una información clara y precisa sobre su diagnóstico². Similarmente, en otro estudio español realizado por Labrador y Bara en 2004, donde se evaluaron a 100 pacientes oncológicos, con diferentes tipos de cáncer, se observó que el 52\% fue informado de forma específica, frente al $48 \%$ que fue informado de manera inespecífica 5 .

En ocasiones, los clínicos omiten la información por miedo a la reacción del paciente o por la actitud paternalista de cuidado hacia él, sin ser conscientes de las repercusiones psicológicas negativas que ello implica, tales como ansiedad y depresión. A esta omisión de la información por parte del personal médico o de los familiares del paciente enfermo se le denomina conspiración o pacto de silencio. La conspiración de silencio sería "el acuerdo implícito o explícito por parte de familiares, amigos y/o profesionales, de alterar la información que se da al paciente con el objetivo de ocultarle el diagnóstico y/o pronóstico y/o gravedad de la situación"6.

En otra investigación española, dirigida por RuizBenítez y Coca (2008), se encontró que solamente el $21 \%$ de los pacientes oncológicos al final de vida conocían su diagnóstico y pronóstico, informado por sus familiares. Y el factor que más peso tenía en los familiares para ocultar la información era el miedo a las repercusiones emocionales negativas derivadas de la comunicación sobre el estado real del paciente?.

Por otra parte, en un estudio donde se analizó el pacto de silencio y las actitudes asociadas en una unidad de cuidados paliativos, se vio que, al momento del ingreso, el $14 \%$ de los pacientes desconocían el diagnóstico y el $71 \%$ desconocía el pronóstico. En cuanto a las actitudes observadas, el $50 \%$ de los pacientes presentaba un mecanismo de negación (negaba, evitaba, no hablaba ni quería saber el diagnóstico o el pronóstico); el 55\% de los familiares quería proteger al paciente y por ello no le comunicaba la información; y el 100\% de los profesionales no tenía dificultad en comunicar el pronóstico. Este estudio mostró la complejidad de la comunicación de información médica al paciente sobre su estado y consideró necesaria una mayor atención por parte de los profesionales de la salud ante estos casos de pacto de silencio por parte de los familiares o de negación por parte del paciente ${ }^{8}$.

Esta omisión de información médica está influida por variables como el miedo a las repercusiones emocionales del paciente y el afán de protegerlo. Pero este fenómeno tiene implicaciones éticas e influye en la práctica clínica, afectando al paciente, a los familiares, al equipo médico y al propio sistema sanitario9. 
Además, en algunas investigaciones se ha visto que esta falta de información puede resultar perjudicial para el funcionamiento psicológico del paciente. Por ejemplo, en un estudio realizado en España, se encontró que el 19,3 \% de las familias de pacientes con gliobastoma multiforme no consideraba pertinente informar al paciente de su pronóstico y se observó que esto afectaba al ajuste psicológico del paciente, evaluado mediante la Escala Hospitalaria de Ansiedad y Depresión (HADS). En concreto, se observó que había un aumento en los niveles de ansiedad del paciente, hasta incluso un mes después del alta hospitalaria. Los pacientes cuyos familiares decidieron un pacto de silencio presentaban niveles psicopatológicos de ansiedad, mientras que los pacientes sin este pacto presentaban niveles de ansiedad por debajo de la media normal ${ }^{10}$.

En otro estudio realizado en México por Reynoso et al., (2008), se evaluaron las principales necesidades de pacientes oncológicos al final de la vida, comparando las respuestas de los propios pacientes y de los médicos. Se exploraron las necesidades de los pacientes en cinco áreas: médica, psicológica, social, económica y espiritual. Se encontró que una de las necesidades más importantes para los pacientes fue conocer toda la información sobre el pronóstico de la enfermedad. Es decir, tener conocimiento acerca de los síntomas que iban a aparecer y del tiempo de vida que les quedaba. Sin embargo, los médicos no le dieron tanto valor a esta necesidad. A pesar de que no hubo grandes variaciones entre las respuestas de las dos muestras, se observaron algunas diferencias que, según los autores, podrían sugerir cambios en los procesos de formación médica con el fin de conseguir una mejor relación médico-paciente ${ }^{11}$.

También se ha visto que los familiares de pacientes con cáncer presentan estas necesidades de información médica y que los síntomas de ansiedad y depresión, medidos mediante la HADS, están relacionados con las necesidades no cubiertas de información acerca de la enfermedad ${ }^{12}$. Además, se ha observado que la información médica acerca de la enfermedad, los síntomas, el tratamiento y los procedimientos quirúrgicos reduce los niveles de ansiedad en padres de niños enfermos ${ }^{13}$.

En el cáncer de mama, se han realizado algunas investigaciones al respecto y se ha encontrado que una de las necesidades de estas pacientes es justamente la demanda de información sobre su diagnóstico, pronóstico y efectos del tratamiento ${ }^{14-18}$. Incluso, se ha mostrado la tendencia a la consulta de páginas web con información médica relevante con el afán de paliar esta necesidad informativa ${ }^{19}$. Además, aunque no todas las mujeres que padecen cáncer de mama quieren tomar un rol activo en cuanto a preguntar sobre su estado ni tomar decisiones médicas, como la aceptación del tratamiento, un gran número de ellas sí quieren participar en las decisiones sobre su tratamiento y por tanto, desean estar informadas de todo el proceso ${ }^{20-23}$.

En este sentido, en un estudio realizado por Jenkins et al., (2001), donde se evaluaron las necesidades de información en una muestra de 2331 pacientes con cáncer de diversos tipos, se encontró que el $87 \%$ quería toda la información posible, tanto buena como mala. Es decir, la mayoría de los pacientes necesitaba conocer el diagnóstico y pronóstico de su enfermedad ${ }^{24}$. En la misma línea, en otro estudio de Palma et al., (2014), donde se entrevistó a 100 pacientes con varios tipos de cáncer, se observó que el 90\% de ellos quería recibir información completa sobre su diagnóstico y el $89 \%$ sobre el pronóstico de su enfermedad ${ }^{25}$.

Con base a esto, algunos autores creen que la decisión de las pacientes con cáncer de mama de no ser informadas puede revertirse a partir de una buena relación médico-paciente ${ }^{26}$. Es decir, es importante trabajar la empatía de los propios médicos hacia los pacientes, puesto que ello puede derivar en una relación más colaborativa y en una mayor implicación del paciente respecto al conocimiento de su enfermedad y en la adherencia al tratamiento médico.

El objetivo de esta investigación fue analizar la relación entre los niveles de información médica sobre el diagnóstico, el pronóstico y el tratamiento con los síntomas de ansiedad y depresión en una muestra de pacientes con cáncer de mama.

\section{Materiales y métodos}

\section{PARTICIPANTES}

Se contó con una muestra de 131 mujeres diagnosticadas con cáncer de mama, provenientes de la Unidad de Oncología de cuatro hospitales públicos de España. 
El tipo de muestreo fue no probabilístico y los criterios de inclusión fueron: que hubiese transcurrido un lapso de tiempo mínimo de un año tras el tratamiento adyuvante, esto debido a que habitualmente se encuentra que el tratamiento con quimioterapia, radioterapia u hormonoterapia se relacionan con síntomas de ansiedad y depresión; se buscó evitar cualquier tipo distorsión en los resultados y falsos positivos en los niveles de ansiedad y depresión; encontrarse en las fases I o II de la enfermedad; aceptar y firmar el consentimiento informado.

Como criterio de exclusión, se eliminaron los casos que no respondieron los cuestionarios en su totalidad y en los cuales se dejaron ítems por responder.

\section{INSTRUMENTOS DE EVALUACIÓN}

Para recabar los datos se utilizó la entrevista clínica semiestructurada realizada por los psicólogos y oncólogos de los cuatro hospitales, en la que se recogieron datos sociodemográficos y clínicos, los niveles de información médica sobre el diagnóstico, pronóstico y tratamiento, así como el grado de comprensión de esta información.

Para ello, se abordaron preguntas específicas de respuesta tipo Likert de 1 a 4 (donde 1 era "no es conocedor" y 4 "totalmente conocedor"), las cuales eran: ¿Del 1 al 4 , en qué medida tiene conocimiento acerca de su diagnóstico? ¿Del 1 al 4, en qué medida tiene conocimiento acerca del tratamiento? ¿Del 1 al 4, en qué medida tiene conocimiento acerca del pronóstico?

Para evaluar el nivel de comprensibilidad de la información médica recibida, se recabaron los datos mediante la pregunta ¿Hasta qué punto cree que fue comprensible la información que le dieron sobre su enfermedad?, con respuesta tipo Likert de 0 a 10, donde o era "nada comprensible" y 10 "totalmente comprensible".

La Escala Hospitalaria de Ansiedad y Depresión $(\text { HADS })^{27}$ adaptada en población española oncológica $^{28}$, la cual consta de 14 ítems con dos sub-escalas de 7 ítems: una de ansiedad y otra de depresión. La suma de las dos sub-escalas corresponde a la puntuación total de la escala, HADS Total, y evalúa el malestar general mediante síntomas tanto de ansiedad como de depresión, como por ejemplo, sensaciones de tensión, miedo, preocupación, pérdida del interés y disfrute en actividades. Se puntúa en una escala tipo Likert de 4 puntos (rango 0-3). El puntaje máximo de cada sub-escala es de 21 y una puntuación de 0 a 7 indica ausencia de ansiedad o depresión, de 8 a 10 indica la necesidad de consideración sintomatológica y más de 11 puntos la presencia de síntomas de ansiedad o depresión. La fiabilidad de la escala, como puede verse en la tabla 1, en la versión adaptada que se utilizó era de 0,85 para la sub-escala de ansiedad y 0,87 para la de depresión y los puntos de corte de 9 para la ansiedad y de 4 para la depresión. En el presente estudio con la muestra evaluada, se obtuvieron índices de fiabilidad de 0,91 para la escala total, de 0,86 para la sub-escala de ansiedad y de 0,88 para la sub-escala de depresión.

Tabla 1. Fiabilidad escala HADS

\begin{tabular}{|l|c|c|c|}
\hline & $\begin{array}{c}\text { HADS } \\
\text { Total }\end{array}$ & $\begin{array}{c}\text { HADS } \\
\text { Ansiedad }\end{array}$ & $\begin{array}{c}\text { HADS } \\
\text { Depresión }\end{array}$ \\
\hline $\begin{array}{l}\text { Fiabilidad } \\
\text { HADS } \\
\text { adaptada a } \\
\text { pacientes } \\
\text { oncológicos }\end{array}$ & 0,85 & 0,87 \\
\hline $\begin{array}{l}\text { Fiabilidad } \\
\text { HADS del } \\
\text { presente } \\
\text { estudio }\end{array}$ & 0,91 & 0,86 & 0,88 \\
\hline
\end{tabular}

Fuente: Autor.

\section{PROCEDIMIENTO}

Las pacientes fueron reclutadas de consultas externas de la Unidad de Oncología de cuatro hospitales públicos de España. El criterio de selección fue no probabilístico y por disponibilidad de la muestra; a las pacientes que acudían a una revisión médica se les preguntaba si querían participar en la investigación, y después de que aceptaran y firmaran el consentimiento informado, se procedió a la recogida de datos, la cual consistió en el pase de pruebas en un único momento de evaluación.

Una vez recogidos los datos, éstos fueron codificados en una base de datos y posteriormente analizados mediante el programa estadístico SPSS 17.

Las variables a estudiar fueron el nivel de conocimiento acerca del diagnóstico de la enfermedad, pronóstico y tratamiento, el nivel de comprensibilidad de la información médica proporcionada y los niveles 
de ansiedad y depresión. Para hacer los análisis estadísticos, se utilizaron estadísticos descriptivos, como la media y porcentajes, y medidas de relación lineal como la correlación de Pearson, con un intervalo de confianza del $95 \%$ y un valor alfa de significancia de 0.05 .

\section{Resultados}

\section{DATOS SOCIODEMOGRÁFICOS Y CLÍNICOS}

De un total inicial de 139 participantes, finalmente las que conformaron la muestra final fueron 131, debido a que 8 de ellas dejaron varios ítems por responder.

En la tabla 2 se evidencian las características sociodemográficas y clínicas más relevantes que fueron tomadas en cuenta en el estudio.

Las puntuaciones de ansiedad y depresión, medidas con la escala HADS adaptada en pacientes oncológicos españoles, fueron de 8.13 (DT= 1.42) para la ansiedad y $5(\mathrm{DT}=1.05)$ para la depresión.

La correlación entre las sub-escalas de ansiedad y depresión fue de $0.671(p<0.01)$, mientras que la correlación entre la sub-escala de ansiedad y la escala total fue de 0.913 ( $p<0.01)$; y para la sub-escala de depresión y la escala total de $0.915(p<0.01)$. Estos datos indican la fuerte relación entre los síntomas de ansiedad y de depresión en los pacientes con cáncer.

\section{NIVELES DE INFORMACIÓN Y SÍNTOMAS DE ANSIEDAD Y DEPRESIÓN}

Los análisis de los resultados mediante la correlación de Pearson, como puede observarse en la tabla 3, informaron de la existencia de relaciones estadísticamente significativas y negativas en el caso de la relación entre el malestar general medido con el HADS y la información sobre el diagnóstico de la enfermedad $(r=-0.247 ; p<0.001)$, la información sobre el tratamiento y efectos secundarios del mismo $(r=-0.240 ; p<0.001)$ y el grado de información comprensible $(r=-0.180 ; p<.005)$. No se obtuvo una correlación estadísticamente significativa entre la escala HADS Total y la información sobre el pronóstico de la enfermedad $(p>0.05)$.

Por otra parte, se encontraron relaciones estadísticamente significativas y negativas en el caso de la relación entre la depresión y la información sobre el diagnóstico de la enfermedad $(r=-0.339$; $p<0.001)$, el pronóstico $(r=-0.238 ; p<.001)$ y el tratamiento y efectos secundarios del mismo $(r=-$ 0.322; $p$ <.001). Es decir, que la falta de información sobre la enfermedad, el pronóstico y el tratamiento se relacionaría con niveles más elevados de depresión.

Sin embargo, las relaciones exploradas acorde con este objetivo no resultaron significativas en el caso de la relación entre la Ansiedad y la información sobre el diagnóstico y el pronóstico de la enfermedad, ni en la información sobre el tratamiento y efectos secundarios $(p>0.05)$.

Por otra parte, la variable de Información comprensible, mostró relaciones significativas y negativas entre esta variable y la Depresión ( $r=-$ $0.242 ; p<0.005)$, pero no significativa en Ansiedad $(r=-0.086 ; p>0.05)$. Estos valores sugieren que la información incomprensible sobre la enfermedad, se relaciona con mayores niveles de Depresión.

\section{Discusión}

Comunicar a un paciente que tiene cáncer es un arduo trabajo, puesto que ponemos en juego la estabilidad emocional de la persona al momento de informarle sobre el diagnóstico. Sin embargo, ocultar o minimizar esta información puede llevar a un peor manejo psicológico por parte del paciente, por el miedo e impotencia que puede generar la situación de incertidumbre. $Y$ todo ello puede repercutir en el bienestar emocional del paciente y promover la aparición de síntomas patológicos de ansiedad y depresión.

En esta investigación se mostró la importancia que tiene la información en el aspecto psicológico de un grupo de mujeres con cáncer de mama, encontrando que las mujeres que tenían menos información sobre su estado presentaban mayores niveles de malestar general medido mediante la escala HADS, refiriendo sensaciones de tensión, miedo, preocupaciones, anhedonia o pérdida de interés.

En concreto, se observó que las pacientes que tenían información más precisa y exacta sobre el diagnóstico de su enfermedad, el pronóstico y los efectos secundarios del tratamiento, y que además, dicha información era comprensible para ellas, presentaban niveles más bajos de depresión que aquellas mujeres que no poseían información sobre estas variables, tenían poca información o 
esta información era incomprensible para ellas. Sin embargo, esta falta de información no se relacionaba con sintomatología ansiosa.

Tabla 2. Datos sociodemográficos y clínicos

\begin{tabular}{|c|c|c|c|}
\hline Característica & $\mathbf{n}$ & $\%$ & Media \\
\hline Edad & & & $\begin{array}{l}55.12 \text { años } \\
\left(\mathrm{DT}^{*}=9.91\right)\end{array}$ \\
\hline \multicolumn{4}{|l|}{ Estado civil } \\
\hline Casada & 90 & 68.7 & \\
\hline Divorciada/separada & 14 & 10.7 & \\
\hline Pareja de hecho & 5 & 3.8 & \\
\hline Soltera & 10 & 7.6 & \\
\hline Viuda & 12 & 9.2 & \\
\hline \multicolumn{4}{|l|}{ Tipo de cirugía } \\
\hline Mastectomía & 58 & 44.3 & \\
\hline Cirugía conservadora & 73 & 55.7 & \\
\hline \multicolumn{4}{|l|}{$\begin{array}{l}\text { Tratamiento adyuvante } \\
\text { recibido }\end{array}$} \\
\hline Quimioterapia & 6 & 4.6 & \\
\hline Radioterapia & 10 & 7.7 & \\
\hline Terapia hormonal & 6 & 4.6 & \\
\hline Quimioterapia + & 28 & 21.5 & \\
\hline \multicolumn{4}{|l|}{ Radioterapia } \\
\hline Quimioterapia + & & & \\
\hline $\begin{array}{l}\text { Radioterapia + Terapia } \\
\text { hormonal } \\
\text { Situación laboral }\end{array}$ & 81 & 61.4 & \\
\hline Trabajadora activa & 60 & 45.8 & \\
\hline Desempleada & 5 & 3.8 & \\
\hline Ama de casa & 30 & 22.9 & \\
\hline En incapacidad temporal & 16 & 12.2 & \\
\hline \multicolumn{4}{|l|}{ por la enfermedad } \\
\hline Jubilada & 20 & 16 & \\
\hline \multicolumn{4}{|l|}{ Nivel de estudios } \\
\hline Sin estudios & 5 & 3.8 & \\
\hline Primaria & 55 & 42 & \\
\hline Bachiller o secundaria & 40 & 30.5 & \\
\hline Diplomado & 12 & 9.2 & \\
\hline Licenciatura & 17 & 13 & \\
\hline Posgrado & 1 & 0.8 & \\
\hline Otros & 1 & 0.8 & \\
\hline Nivel de ansiedad & & & 8.13 \\
\hline Nivel de depresión & & & 5 \\
\hline
\end{tabular}

DT*: Desviación típica/estándar

Fuente: Autor.

Estos resultados corroboran los resultados de estudios previos, como los realizados en España por
Rodríguez-Castillo, 2015, y Díaz-Cordobés et al., 2012; en Australia, por Oberoi et al., 2016; y en Burkina Faso por Kabore et al., 2016, donde se postula que el pacto de silencio y el hecho de proporcionar información médica no precisa ni concreta a los pacientes, tiene repercusiones en el buen funcionamiento psicológico de los mismos, pudiendo presentarse incertidumbre, miedo, preocupación, temor, tristeza, anhedonia y apatía, que pueden derivar a la aparición de síntomas de ansiedad y depresión 9,10,12,13. En este sentido, los autores postulan que la falta de información médica que tiene el paciente sobre su enfermedad está relacionada con los síntomas comentados anteriormente.

Sin embargo, aunque previamente se ha tratado de analizar las necesidades de información médica que presentan las pacientes con cáncer de mama ${ }^{14-22}$, apenas hay investigaciones que relacionen estas necesidades y las afectaciones en el bienestar emocional. Es por ello que el presente estudio aporta datos novedosos respecto a la relación entre la falta de información médica y la aparición de sintomatología depresiva en esta enfermedad en concreto.

Algunas de las limitaciones encontradas en el presente estudio que pudieran estar influyendo en los resultados son, por una parte, que no se tuvo en cuenta el nivel educativo de las pacientes y algunos autores postulan que esta variable, en concreto un alto nivel educativo, está relacionada con un menor distres, menores niveles de depresión y menores problemas interpersonales, como podría ser el caso de los problemas de comunicación entre médico y paciente ${ }^{29-31}$.

Además, la presente muestra se define como asintomática en relación a los síntomas de depresión, con una puntuación media de 5 en la escala HADS (recordemos que para ser considerados síntomas clínicamente relevantes según la escala HADS, la puntuación media debe de ser 8 o más); y con sintomatología ansiosa moderada (puntuación de 8.13). Esto debe de tomarse en cuenta, ya que para poder dilucidar mejor los resultados, habría que contar con una muestra que presente tanto síntomas de depresión como de ansiedad.

Por otro lado, tampoco se tuvo en cuenta el tiempo de diagnóstico. Esta es una variable que puede tener un papel importante, ya que como algunos autores indican, el bienestar emocional va disminuyendo a medida que avanza la enfermedad, apareciendo 
perfiles sintomatológicos diferenciales según el momento transcurrido desde el diagnóstico ${ }^{29,32-34}$. Este dato podría estar interfiriendo en los resultados, por ejemplo, en el hecho de que haya relaciones estadísticamente significativas entre la depresión y la información sobre la enfermedad, el tratamiento y el pronóstico, pero no entre la ansiedad y estas variables.

Tabla 3. Información sobre la enfermedad, ansiedad y depresión

\begin{tabular}{|c|c|c|c|c|}
\hline & $\begin{array}{l}\text { Información } \\
\text { diagnóstico }\end{array}$ & $\begin{array}{c}\text { Información } \\
\text { Tratamiento y efectos } \\
\text { secundarios }\end{array}$ & $\begin{array}{l}\text { Información } \\
\text { pronóstico }\end{array}$ & $\begin{array}{l}\text { Información } \\
\text { comprensible }\end{array}$ \\
\hline HADS Total & $\begin{array}{c}r=-0,247 \\
p<0.001\end{array}$ & $\begin{array}{c}r=-0,240 \\
p<0,001\end{array}$ & $\begin{array}{c}r=-0,158 \\
p>0,05\end{array}$ & $\begin{array}{c}r=-0,180 \\
p<0,005\end{array}$ \\
\hline HADS Ansiedad & $\begin{array}{c}r=-0,110 \\
p>0,05\end{array}$ & $\begin{array}{c}r=-0,114 \\
p>0,05\end{array}$ & $\begin{array}{c}r=-0,050 \\
p>0,05\end{array}$ & $\begin{array}{c}r=-, 086 \\
p>0,05\end{array}$ \\
\hline HADS Depresión & $\begin{array}{l}r=-0,339 \\
p<0,001\end{array}$ & $\begin{array}{c}r=-0,322 \\
p<0,001\end{array}$ & $\begin{array}{c}r=-0,238 \\
p<0,001\end{array}$ & $\begin{array}{c}r=-0,242 \\
p<0,005\end{array}$ \\
\hline
\end{tabular}

Fuente: Autor.

Por último, es importante proporcionar herramientas de manejo de información médica y habilidades de comunicación a los profesionales en beneficio de la calidad de vida de los pacientes, ya que se puede observar mediante investigaciones como la realizada por Rodríguez-Castillo (2015) y en este estudio, que el hecho de omitir la información al paciente, el pacto de silencio o el proveer de escasa información, puede llevar a que los pacientes manifiesten reacciones emocionales como temor, miedo, preocupación, apatía, anhedonia; pudiendo dar lugar a síntomas de ansiedad y depresión.

\section{CONCLUSIONES}

La información que reciben las pacientes con cáncer de mama sobre su enfermedad, el pronóstico y el tratamiento médico influye en su bienestar emocional, presentándose mayores niveles de depresión en las pacientes que tienen escasa información, o que consideran que la información proporcionada por el equipo médico es incomprensible.

\section{COMITÉ DE ÉtICA}

Esta investigación fue aceptada por parte del Comité de ética de la Universidad de Valencia, siguiendo los estándares de la declaración de Helsinki de 1964 de investigación en humanos.

\section{CONFLicto de Intereses}

No existen conflictos de interés

\section{FinANCIACIÓN}

No hay financiación.

\section{Referencias Bibliográficas}

1. Thorne S, Oliffe JL, Stajduhar KI, Oglov V, Kim-Sing C, Hislop TG. Poor communication in cancer care: patient perspectives on what it is and what to do about it. Cancer nurs. 2013; 36(6):445-53.

2. Peiró G, Corbellas C, Camps C, Sánchez PT, Godes M, D’Ambra $\mathrm{M}$, et al. Estudio descriptivo sobre el proceso de comunicar el diagnóstico y el pronóstico en oncología. Psicooncología. 2006;3(1):9-17.

3. Trill MD. Influencia de la cultura en la experiencia del cáncer. Psicooncología. 2003;0(1): 39-48.

4. López B, Tomás M, Escolar A. Percepción y satisfacción respecto a la información recibida en el proceso de atención médica en pacientes con cáncer de próstata. Actas Urol Esp. 2016;40(2):88-95.

5. Labrador FJ, Bara E. La información del diagnóstico a pacientes de cáncer. Psicooncología. 2004;1(1):51-66.

6. Barbero J. El derecho del paciente a la información: el arte de comunicar. An Sist Sanit Navar. 2006;29(3):19-27.

7. Ruiz-Benítez MA, Coca MC. El pacto de silencio en los familiares de los pacientes oncológicos terminales. Psicooncología. 2008;5(1):53-69.

8. Bermejo JC, Villacieros M, Carabias R, Sánchez E, Díaz-Albo B. Conspiración del silencio en familiares y pacientes al final de la vida ingresados en una unidad de cuidados paliativos: nivel de información y actitudes observadas. Medicina paliativa. 2013;20(2):49-59.

9. Rodríguez-Castillo C. Conspiración del silencio. Revista Española de Comunicación en Salud. 2015;6(2)213-21.

10. Díaz-Cordobés JL, Barcia JA, Gallego-Sánchez JM, Barreto P. Conspiración de silencio y malestar emocional en pacientes diagnosticados de glioblastoma multiforme. Psicooncología. 2012;9(1):151-9

11. Reynoso E, Alazraki O, González-Maraña M, Alvarado S, Pulidol MA. Evaluación de las necesidades de pacientes oncológicos en etapa terminal (a través del instrumento ENPOET): comparación entre muestras. Psicooncología. 2008;5(1):39-52

12. Oberoi D, White V, Jefford M, Giles GG, Bolton D, David I, et al. Caregivers' information needs and their 'experiences of care' during treatment are associated with elevated anxiety and depression: a cross-sectional study of the caregivers of renal cancer survivors. Support Care Cancer. 2016;24(10):4177-86.

13. Kabore FA, Traore IS, Ouédraogo D, Kabre Y, Traore IA, Sanou J, et 
al. Preoperative medical information reduces anxiety of parents of children who underwent surgery. Prospective randomized study. Anesth Analg. 2016;123(3S_Suppl):602-3.

14. Ferro T, Prades J. Necesidades de información en el cáncer de mama y de atención en la supervivencia. Barcelona: FECMA; 2013. $67 \mathrm{p}$.

15. Koopman C, Angell K, Turner-Cobb JM, Kreshka MA, Donnelly P, McCoy R, et al. Distress, coping, and social support among rural women recently diagnosed with primary breast cancer. Breast J. 2001;7(1):25-33.

16. Landmark BT, Bøhler A, Loberg K, Wahl AK. Women with newly diagnosed breast cancer and their perceptions of needs in health-care context. J Clin Nurs. 2008;17(7b):192-200.

17. Ussher JM, Perz J, Gilbert E. Information needs associated with changes to sexual well-being after breast cancer. J Adv Nurs. 2013;69(2):327-37.

18. Wilson SE, Andersen MR, Meischke H. Meeting the needs of rural breast cancer survivors. What still needs to be done?. J Womens Health Gend Based Med. 2000;9(6):667-77.

19. Sacks AA, Hernando SP, Aguilar PS, Vega EF, Fernández RM. Necesidades de información y uso de Internet en pacientes con cáncer de mama en España. Gac Sanit. 2013;27(3):241-7.

20. Bilodeau BA, Degner LF. Information needs, sources of information, and decisional roles in women with breast cancer. Oncol Nurs Forum. 1996;23(4):691-6.

21. Degner LF, Kristjanson LJ, Bowman D, Sloan JA, Carriere KC, O'Neil J, et al. Information needs and decisional preferences in women with breast cancer. Jama. 1997;277(18):1485-92.

22. Rees CE, Bath PA. The information needs and source preferences of women with breast cancer and their family members: a review of the literature published between 1988 and 1998. J Adv Nurs. 2000;31(4):833-41.

23. Martín-Fernández R, Abt-Sacks A, Perestelo-Perez L, SerranoAguilar P. Actitudes de las mujeres diagnosticadas de cáncer de mama frente a la toma de decisiones compartida. Rev Esp Salud Pública. 2013;87(1):59-72.

24. Jenkins V, Fallowfield L, Saul J. Information needs of patients with cancer: results from a large study in UK cancer centres. $\mathrm{Br}$ Cancer. 2001;84(1):48-51.
25. Palma A, Cartes F, González M, et al. ¿Cuánta información desean recibir y cómo prefieren tomar sus decisiones pacientes con cáncer avanzado atendidos en una Unidad del Programa Nacional de Dolor y Cuidados Paliativos en Chile?. Rev Med Chil. 2014;142(1):48-54.

26. Piñeiro Fernández Justo. ¿debe el paciente conocer que tiene un cáncer?: Nuestra experiencia en las pacientes con cáncer de mama. Rev Cubana Cir [Internet]. 2004 Mar [citado 2017 Sep 18] ; 43 1 ): . Disponible en: http://scielo.sld.cu/scielo.php?script=sci arttext\&pid $=$ S0034-74932004000100007\&lng=es.Zigmon A, Snaith R. The Hospital Depression and Anxiety Scale. Acta psychriat scand. 1983;67:361-370.

27. Zigmond A, Snaith R. The Hospital Depression and Anxiety Scale. Acta psychriat scand. 1983;67:361-70

28. López-Roig S, Cantero Terol MC, Pastor MA, Massuti B, Rodríguez-Marín J, Leyda JI, et al. Ansiedad y depresión. Validación de la escala HAD en pacientes oncológicos. Rev Psicol Salud. 2000;2(12):127-57.

29. Carver C, Smith R, Antoni M, Petronis V, Weiss S, Derhagopian R. Optimistic personality and psychosocial well-being during treatment predict psychosocial well-being among long-term survivors of breast cancer. Health Psychol. 2005;24(5):508-16.

30. Compas B, Luecken L. Psychological adjustment to breast cancer. Curr. Dir. Psychol. Sci. 2002;11(3):111-4

31. Schover L, Yetman L, Tuason L, Meiser E, Esselsfym C, Hermann $\mathrm{R}$, et al. Mastectomy and breast reconstruction. A comparison of their effects on psychosocial adjustment, body image, and sexuality. Cancer. 1995;75(1):54-64

32. 8. Ganz P, Desmond K, Leedham B, Rowland J, Meyerowitz B, Belin T. Quality of life in long-term, disease-free survivors of breast cancer: a follow-up study. J Natl Cancer Inst [Internet]. 2002 [Citado 2016 Dic 15];94(1):39-49.

33. 9. Hanson M, Suman V, Rumans T, Dose A, Taylor M, Novotny P, et al. Physical, psychological and social well-being of women with breast cancer: the influence of disease phase. Psychooncology [Internet]. 2000 [Citado 2016 Dic 15];9:221-31.

34. Urcuyo K, Boyers A, Carver C, Antoni M. Finding benefit in breast cancer: Relations with personality, coping, and concurrent well-being. Health Psychol. 2005;20(2):175-92. 